\title{
Rechtsgeschichte
}

http://www.rg-rechtsgeschichte.de/rg4

$\operatorname{Rg} 2004$

$239-242$

Zitiervorschlag: Rechtsgeschichte Rg 4 (2004)

http://dx.doi.org/10.12946/rg04/239-242

\section{Ralf Michaels}

\section{Fünf Minuten Rechtsvergleichung}


gere Ausbeute: Die Frage nach der Funktion der Rechtsstaatsdebatte im Dritten Reich setzt eine historische Problemdiagnose voraus. Es ginge darum, festzustellen, mit welchen konkreten Problemen die Staatsrechtswissenschaft jener Zeit konfrontiert war und inwiefern bestimmte Rechtsstaatskonzepte eine zweckgerechte Lösung jener Probleme versprachen und andere nicht. Ohne eine erschöpfende Analyse des poli- tischen und gesellschaftlichen Zusammenhanges, ohne eine vertiefte Auseinandersetzung mit dem biographischen Hintergrund der Beteiligten kann eine solche Diagnose nicht gelingen. Ohne Sichtung, Erschließung und Kategorisierung des Quellenmaterials allerdings auch nicht. Diese Leistung hat Hilger erbracht.

Natascha Doll

\section{Fünf Minuten Rechtsvergleichung*}

Radbruch ist unser aller Lieblingsrechtsphilosoph, also auch der des Rechtsvergleichers. Die Liebe gründet sich vor allem natürlich auf die berühmte Radbruch'sche Formel vom gesetzlichen Unrecht und die daran hängende wundersame Legende von der Bekehrung Radbruchs vom Saulus des Rechtspositivismus zum Paulus des Naturrechts, vom alttestamentarischen Schrift- und Buchstabengläubigen zum neutestamentarischen Gläubigen an den Geist des Rechts. Radbruchs wichtigster Beitrag zur Rechtsvergleichung scheint dagegen in der von Zweigert und Kötz in ihrem Rechtsvergleichungslehrbuch benutzten Aussage zu bestehen, dass Wissenschaften, die sich mit ihrer eigenen Methode zu beschäftigen haben, kranke Wissenschaften $\operatorname{sind}^{\mathbf{I}}$ - und die war ursprünglich nicht einmal auf die Rechtsvergleichung gemünzt. Dem Eindruck, dass Radbruch mit Rechtsvergleichung nichts zu tun gehabt habe, will nun Heinrich Scholler als Herausgeber des einschlägigen Bandes in der Gesamtausgabe und Verfasser einer Studie entgegenwirken: Radbruch war danach nicht nur Rechtsvergleicher, sondern hat sich, so der Waschzettel zur Studie, sogar »bahnbrechend mit den zentralen Fragen der Rechtsvergleichung beschäftigt « - er hat nämlich eine neue Disziplin begründet, »die im angloamerikanischen Recht als >Comparative Legal Culture bezeichnet wird « und die Scholler mal als Rechtskulturvergleich, mal als vergleichende Rechtskulturwissenschaft bezeichnet. Und es kommt noch besser: Seine rechtsvergleichende Beschäftigung mit dem englischen Recht, insbesondere der equity als Korrektur des Rechts im Namen der Billigkeit, war sogar »maßgebend « für die Lehre vom gesetzlichen Unrecht.

Das klingt interessant, und so greift man zunächst zu Schollers eigener Studie. Bald stellt sich allerdings heraus, dass es sich dabei eher um die von ihm selbst im Band der Gesamtausgabe bereits für eigentlich nötig befundene längere Einleitung zu jenem Band handelt, als um eine eigenständige Arbeit. Tatsächlich übernimmt das Büchlein die ersten vierzig Seiten (zur Rechtsvergleichung bei Radbruch im Allgemeinen) fast wörtlich und vollständig aus dem Vorwort, teilweise umgestellt, aber unter Übernahme sogar der Tippfehler. Auch sonst findet sich wenig außer Paraphrasierungen der Werke Radbruchs,

\footnotetext{
* Heinrich Scholler, Die Rechtsvergleichung bei Gustav Radbruch und seine Lehre vom überpositiven Recht (Schriften zur Rechtstheorie, 2 I0), Berlin: Duncker \& Humblot 2002, I2I S., ISBN 3-428-I0904-X; Gustav RadBRuch, Rechtsvergleichende Schriften, bearbeitet von HeINRICH SCHOLLER (Gustav Radbruch Gesamtausgabe, Bd. I 5 ), Heidelberg:
}

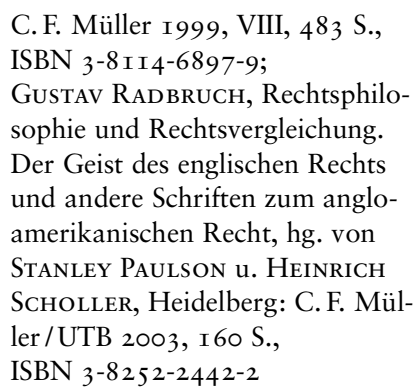

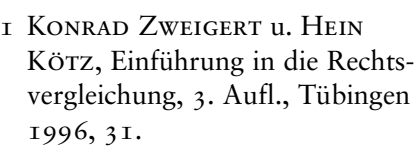


und das Wenige ist auch nicht recht neu: fünf Seiten zur allgemeinen Geschichte der Rechtsvergleichung (26-3I), vier Seiten zu John Austin (63-66), und so weiter. Man blättert mit wachsendem Verdruss, wundert sich über Fehlinformationen (Joseph Story habe den Begriff des »international private law» geprägt, richtig: " private international law «); »die letzte systematische Darstellung des common law nach Glanvill « stamme von Bracton, 49 Fn. I92 (was ist nur mit Blackstone?), Belangloses (Austin war Nachbar von Bentham, James Mill dagegen wohnte nur » in nächster Nähe «, 64), Umständliches (»Arthur Kaufmann hat mit Recht darauf hingewiesen, dass es von großer Bedeutung war, dass Erik Wolf ihn [nur wen? Radbruch?] unter die großen deutschen Rechtsdenker ... eingereiht hatte «, 64 Fn. 265), reihenweise falsch geschriebene Namen (»Hattenauer«, "Yutema «, »Seldon «) und eine ärgerliche Anzahl entstellender Rechtschreibfehler, insbesondere in den englischen Zitaten, sowie eine Reihe teilweise geradezu skurriler Kurzbiographien erwähnter Berühmtheiten (»Sir Matthew Hale, I609-1676. Einer der größten Gelehrten des englischen Rechts [common law], bekannt vor allem durch seine Unparteilichkeit im englischen Bürgerkrieg [I642-I65I] «, 42 Fn. I60).

Und die These von der equity als Basis für das übergesetzliche Recht? Wir erfahren, dass einerseits die Stellung der equity bei Radbruch gar nicht überaus viel Raum in Anspruch nimmt (47), andererseits aber Kaufmann und Paulson schon gezeigt haben, dass der Wandel vom Rechtspositivisten zum Naturrechtler in Wirklichkeit gar kein Wandel war (95 ff.). Anderswo mutmaßt Scholler, wenn Radbruch 1936 in einem Aufsatz zur Rechtssicherheit in England vom Recht zur Revolution gegen ein ungerechtes Recht schreibe, dringe er wohl aus politischen
Gründen bis zum Widerstandsrecht vor - und ist dann verwundert, dass Radbruch den Artikel mit einem gegensätzlichen Zitat schließt: »Vaut mieux vivre là où rien n'est légal que là où tout est légal « (73, Zitat aus dem Original berichtigt).

Also muss man doch bei Radbruch selbst nachlesen. Der Band aus der Gesamtausgabe enthält einerseits, unvermeidlicherweise, nicht alle Aufsätze Radbruchs zur Rechtsvergleichung, so sind etwa die Schriften zu Feuerbach als Rechtsvergleicher im Feuerbach gewidmeten Band 6 der Gesamtausgabe gelandet. ${ }^{2}$ Andererseits finden sich auch Beiträge zum Völkerrecht, eine Sammlung von Buchbesprechungen und Zeitungsartikeln sowie, im Anschluss an die Programmschrift von I9I9 »Ihr jungen Juristen!« (75 ff.), ein zwar nicht rechtsvergleichendes, aber dennoch hochinteressantes Stück Wissenschaftsgeschichte: der polemische (»Was weiß ein revolutionärer Kriminalist von Interpolationenforschung? «) Austausch zwischen dem Sozialisten Radbruch und dem Konservativen Beseler über die Frage, ob das römische Recht zur Studentenausbildung und zur Rechtfertigung des deutschen Durchmarschs durch Belgien I9I 4 geeignet sei (93 ff., 4I 5 ff.)! (Beseler: zweimal ja, Radbruch: zweimal nein). Aber das Schwergewicht liegt doch auf den Arbeiten zur Rechtsvergleichung im weiteren Sinne: Darunter sind der frühe, kurze und recht berühmte Aufsatz »Über die Methode der Rechtsvergleichung " ( 52 ff.), in dem Radbruch als tertium comparationis das Rechtsideal einführt und die Rechtsvergleichung als Sozialwissenschaft, nicht dogmatische Wissenschaft sehen will. Andere Beiträge sind dem Strafrecht gewidmet: vier strafrechtsvergleichende Beiträge für die monumentale sechzehnbändige »Vergleichende Darstellung des deutschen und ausländischen Strafrechts « und eine Einführung in das indische

2 Gustav Radbruch, Feuerbach, bearbeitet von Gerhard Haney, Heidelberg 1997. 
Strafgesetzbuch. Daneben liegt ein Hauptinteresse im angloamerikanischen Recht - ein natürliches Objekt für Radbruch angesichts seines einjährigen Aufenthaltes I935 in Oxford: ${ }^{3}$ Die wichtigsten aufgenommenen Texte in diesem Bereich sind die nach dem Zweiten Weltkrieg erschienene Schrift »Der Geist des englischen Rechts « $(25 \mathrm{ff}$.$) , in der Radbruch das englische$ Recht in höchsten Tönen lobt und nur gegenüber dem Strafrecht Bedenken äußert, ein früher entstandener Aufsatz zur angloamerikanischen Rechtsphilosophie in englischer und französischer Fassung (Anglo-American Jurisprudence through Continental Eyes, 250 ff.) sowie, in französischer Sprache, der schon erwähnte Aufsatz von 1936 zur »sécurité en droit d'après la théorie anglaise «, der im »Geist des englischen Rechts « weitgehend übernommen wird (28I ff., $50 \mathrm{ff}$.$) .$

Hier lässt sich nun die These vom Einfluss des common law auf die Formel vom übergesetzlichen Recht nachprüfen. Und es zeigt sich, dass Radbruch durchaus bewusst ist, wie die equity im englischen Recht längst ebenso formal ist und der Rechtssicherheit dient wie das common law im engeren Sinne (47), sie also zur eigentlichen Billigkeitskorrektur des Rechts nicht taugt. Auch das Recht zur Revolution gegen ungerechtes Recht taucht bei Radbruch nur in der Darstellung Benthams auf, der - getreu seinem utilitaristischen Grundansatz - ein solches Recht bejaht, wenn die Revolution mehr nutzt als schadet ( 55 f., 29I). Tatsächlich scheint Radbruch durchaus auch noch 1936 die Rechtssicherheit nicht nur für wichtig zu halten, sondern gerade umgekehrt als Bollwerk gegen Unrecht hochzuhalten. So heißt es in dem Aufsatz von I936 noch recht allgemein, allerdings schon mit offensichtlicher Missbilligung: »Aujourd'hui des grandes parties de l'humanité pensent pouvoir construire leur univers juridique en dehors de la condition de sécurité en se fondant simplement sur le principe du bien commun, différemment interprété« (292). Dass der anfangs sehr allgemeine Satz (jedenfalls auch) auf das Dritte Reich gemünzt ist, zeigt sich daran, dass seiner Übernahme in den "Geist des englischen Rechts « I946/47 ein weiterer Satz folgt: "Diese Auffassung hat einen furchtbaren Zusammenbruch erlitten « (57). Radbruch sieht es also gar nicht so anders als später etwa Ingo Müller und Bernd Rüthers: Nicht die getreue Anwendung des geltenden Rechts, sondern der freie Umgang mit der Rechtsidee hat das Nazi-Unrecht ermöglicht das englische Recht ist (jedenfalls insoweit) überlegen, nicht weil es weniger, sondern weil es der Rechtssicherheit und dem Recht mehr traut als dem Gewissen des Einzelnen. Noch eine zweite bedeutsame Änderung findet sich allerdings im »Geist des englischen Rechts «: der Hinweis, dass Rechtssicherheit bezüglich des beweglichen englischen common law etwas ganz anderes ist als bezüglich des statischen Gesetzes, weil das common law eine Art natürliche Richtigkeitsgewähr garantiert, die beim auf autoritärem Befehl beruhenden Gesetz fehlt. Hier wäre also ein Ansatz zur Lehre vom Unrechts-Recht zu erkennen wenn sich Radbruch den nicht erst von einem Rezensenten der ersten Auflage (Erdsiek) hätte hineinschreiben lassen müssen (die Rezension ist wörtlich übernommen, 57-59). Den Ungehorsam gegenüber dem Gesetz jedenfalls kann Radbruch in England kaum kennen gelernt haben.

Was soll man abschließend sagen? Schollers eigenes Buch braucht man neben Radbruchs Schriften zur Rechtsvergleichung nicht. Diese wiederum sind interessant zum Verständnis seines eigenen Denkens und als Dokumentation; seinen Beitrag zur Rechtsvergleichung dagegen vermag ich nicht als so bedeutsam einzuschät-

\footnotetext{
3 Carola Vulpius, Gustav Radbruch in Oxford, Heidelberg I995; JoHN BELL, Wolfgang Friedmann (I907-I972), with an excursus on Gustav Radbruch (I878-I94I), in: Jurists Uprooted: German-Speaking Emigré Lawyers in Twentieth Century Britain, hg. v. JaCK BRATSON u. REINHARD ZimMERMANN, Oxford (im Erscheinen), 827-855, $848 \mathrm{ff}$.
} 
zen. Wissenschaftsgeschichtlich am interessantesten ist noch die Einführung des Rechtsideals als Vergleichsmaßstab, ein Gedanke, der heute als Effizienz im comparative law and economics wiederkehrt. Dass daneben Radbruch die »Comparative Legal Culture « (die übrigens entgegen Schollers Ansicht im angloamerikanischen Recht als Begriff jedenfalls im Singular praktisch nicht vorkommt) erfunden habe, erscheint etwas hoch gegriffen: Darstellungen fremder Rechts-»Kultur ", ebenso wie die Idee des Zusammenhangs zwischen Kultur und Recht, gehen mindestens bis auf Montesquieu zurück. Der »Geist des englischen Rechts « selbst reiht sich so schließlich in eine Serie von Studien deutscher Emigranten (Pringsheim, Kantorowicz) zum englischen Recht ein, die die Begeisterung für das als so andersartig empfundene und offenbar so demokratische Recht des Gastlandes gerade im Ge- gensatz zum Unrechtsstaat Nazideutschland erspüren lassen (und sicher auch deshalb im Nachkriegsdeutschland so populär wurden). Aber im Rückblick nimmt Radbruch doch im Überschwang die Selbstbeschreibung der englischen Juristen, etwa bezüglich der Abwehr des römischen Rechts, der intrinsischen Gerechtigkeit des common law, der Bedeutungslosigkeit von statutes im Vergleich zum Fallrecht, ein wenig zu sehr für bare Münze, und seine Essentialisierung eines englischen Volksgeistes ist jedenfalls aus heutiger Sicht problematisch. Dass Radbruchs rechtsvergleichende Schriften die Welt des Rechts letztlich weniger bewegt haben als seine Formel vom gesetzlichen Unrecht, ist daher vielleicht kein Zufall.

\section{Storia del diritto senza storici del diritto*}

Sembra che lei lo senta. Gira la testa verso est, verso di lui, là dove il sole è appena spuntato nel cielo; $e$ quando la macchina si muove, china leggermente la testa come in segno di riconoscenza. Oltre il ponte, la croce bianca della Svizzera. La luce del mattino brilla sul Reno...

Charlie era al sicuro. March alzò gli occhi verso il sole e seppe ... lo seppe con certezza assoluta**

L'atteggiamento tenuto dalla Svizzera e soprattutto dalla sua piazza finanziaria a partire dall'avvento del nazismo in Germania e durante la Seconda Guerra Mondiale è improvvisamente diventato oggetto, nel corso del I996, di aspre polemiche internazionali e di attacchi provenienti da vari Paesi, in particolare dagli Stati Uniti
d'America. Non che non ci fossero state fino a quel momento voci critiche sia nella storiografia che negli ambienti politici in merito a questo tema, tuttavia - complice il velo anestetico che la Guerra fredda stendeva su tutto questo genere di discussioni - era fino ad allora mancato un vero e proprio dibattito interno sulla politica svizzera di quel periodo. La virulenza delle polemiche scatenate nel 1996 ha improvvisamente costretto le élites economiche e politiche elvetiche ad un salutare esame di coscienza, pur non esente da scomposte cadute di tono, che ha portato finalmente a riconoscere la legittimità delle pretese patrimoniali di moltissime vittime dirette ed indirette del nazismo, fino ad allora cinicamente

\footnotetext{
* Die Schweiz, der Nationalsozialismus und der Zweite Weltkrieg. Schlussbericht der Unabhängigen Expertenkommission Schweiz Zweiter Weltkrieg, Zürich: Pendo 2002, 6I9 S., ISBN 3-8 5842-60I-6

* Cit. da Fatherland di RoberT

HARris, trad. ital. a cura di Roberta RAMBELLI, I992.
} 